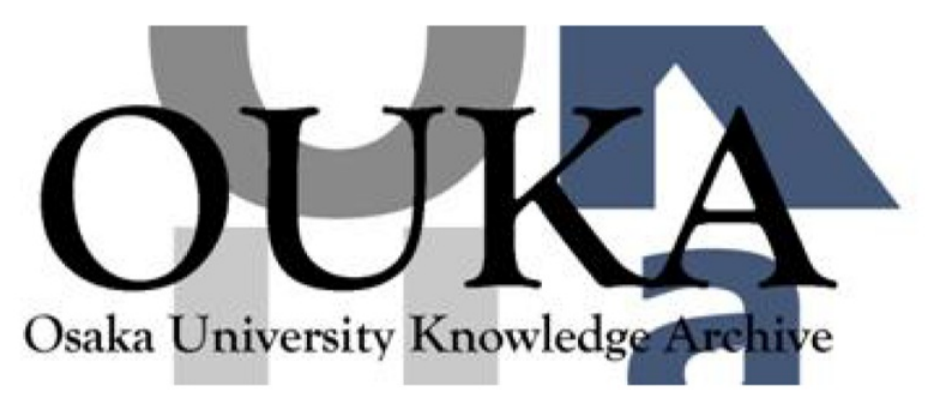

\begin{tabular}{|c|l|}
\hline Title & $\begin{array}{l}\text { Anisotropy of the electro-optic Kerr effect in } \\
\text { polymer-stabilized blue phases }\end{array}$ \\
\hline Author(s) & $\begin{array}{l}\text { Kawata, Yuto; Yoshida, Hiroyuki; Tanaka, Shu et } \\
\text { al. }\end{array}$ \\
\hline Citation & $\begin{array}{l}\text { Physical Review E - Statistical, Nonlinear, and } \\
\text { Soft Matter Physics. 91(2) p.022503-p.022503 }\end{array}$ \\
\hline Issue Date & $2015-02-27$ \\
\hline oaire:version & VoR \\
\hline URL & https://hdl. handle. net/11094/75691 \\
\hline rights & $\begin{array}{l}\text { Copyright (2015) by the American Physical } \\
\text { Society }\end{array}$ \\
\hline Note & \\
\hline
\end{tabular}

Osaka University Knowledge Archive : OUKA

https://ir. Library. osaka-u. ac. jp/

Osaka University 


\title{
Anisotropy of the electro-optic Kerr effect in polymer-stabilized blue phases
}

\author{
Yuto Kawata, Hiroyuki Yoshida, ${ }^{*}$ Shu Tanaka, Anucha Konkanok, and Masanori Ozaki \\ Division of Electrical, Electronic and Information Engineering, Graduate School of Engineering, Osaka University, \\ 2-1 Yamada-oka, Suita, Osaka 565-0871, Japan \\ Hirotsugu Kikuchi \\ Institute for Materials Chemistry and Engineering, Kyushu University, Kasuga, Fukuoka 816-8580, Japan
}

(Received 3 December 2014; published 27 February 2015)

\begin{abstract}
Liquid crystalline polymer stabilized blue phases (PSBPs) are candidate materials for next generation electrooptic switching devices because they form a self-organized three-dimensional periodic structure and exhibit a fast response time of submillisecond order. Considering the crystallographic structures of PSBPs, it is intuitive to believe that the electro-optic effect would depend on the direction of the applied electric field; however, this relationship has not yet been investigated. In this study, we prepared two kinds of samples in which the (110) and (200) planes were oriented parallel to the substrates, and investigated the electro-optic Kerr effect as a field was applied between the two substrates. The two samples exhibited differing behaviors, with the Kerr coefficient of the (110)-oriented sample being larger by $20 \%$ than that of the (200)-oriented sample. These results imply that the electro-optic Kerr effect of PSBPs is not isotropic but anisotropic, just like cubic optical crystals.
\end{abstract}

DOI: 10.1103/PhysRevE.91.022503

PACS number(s): 61.30.Mp, 61.30.Cz, 61.30.Gd, 78.15.+e

\section{INTRODUCTION}

Liquid crystalline cholesteric blue phases (BPs) typically appear between the cholesteric phase and the isotropic liquid in a chiral liquid crystal [1]. The cubic orientational order exhibited by BPs I and II make them interesting both as subjects of soft matter physics and as candidate materials for next generation electro-optic applications [2,3]. For practical applications, BP I is usually polymer stabilized, i.e., a small amount of polymer $(\sim 10 \mathrm{wt} \%)$ is added to the host chiral nematic liquid crystal and is polymerized in situ in either BP I or II. This procedure stabilizes the BP structure such that the $\mathrm{BP}$ is observed for over $60^{\circ} \mathrm{C}$, including room temperature [4]. In polymer-stabilized blue phases (PSBPs), a polymer network is formed templating the array of disclinations that exist in the BP lattice, and prevents the superstructure from being destroyed by external stimuli such as heat or electrical fields [5]. However, the liquid crystal molecules within the lattice are still able to respond to external stimuli and reorient along an electric field. This gives rise to a quadratic electro-optic effect known as the Kerr effect, where the induced birefringence increases in proportion to the square of the applied electric field, i.e.,

$$
\Delta n=\lambda K E^{2}
$$

where $\lambda$ is probe wavelength, $K$ is Kerr coefficient, and $E$ is applied electric field [6]. The Kerr coefficient is considered as one of the most crucial parameters of PSBPs for practical applications, as it determines the driving voltage of the sample. Mainly two approaches are being made in an effort to improve the Kerr coefficient: to improve the material characteristics, and to optimize the blue phase structure. The first approach is mostly chemistry oriented, such as developing liquid crystal molecules with large dielectric anisotropies [7-12]. The second approach involves adjusting the lattice parameter

\footnotetext{
*yoshida@eei.eng.osaka-u.ac.jp
}

or changing the structure of blue phases for improved performance $[13,14]$. In our recent study, we have shown that the Kerr coefficient of PSBPs can vary by more than $10 \%$ when the lattice structure is varied from cubic to tetragonal [14].

Despite the numerous efforts that have been poured into improving the Kerr coefficient of BPs, most studies to date have treated the Kerr coefficient as a scalar property that is independent of the lattice orientation. It is known that for optical crystals with cubic symmetry, the Kerr coefficient is a tensor that is described using three-independent coefficients [15]. However, while signs of field-induced biaxiality has been noted for BP I samples with an electric field applied in the [110] direction [16], and the importance of treating the Kerr coefficient as a tensor has been mentioned in some papers [17,18], quantitative measurements have not been performed. Moreover, PSBPs, in which the lattice structure is fixed, remain completely uninvestigated. It is therefore important to clarify whether the Kerr coefficient of BPs is isotropic or anisotropic.

In this study, we prepared two kinds of PSBP samples in which different BP I lattice planes [(200) and (110) planes] are oriented parallel to the substrates, and compared the Kerr effect as a field was applied between the substrates [hereafter we refer to the two samples as (200)- and (110)-oriented samples: note also that we use (200) instead of (100) as it is the plane from which Bragg reflection occurs in BP I]. It was indeed found that the Kerr coefficient and saturation behavior was different depending on the lattice orientation.

\section{EXPERIMENTAL PROCEDURE}

The PSBP material we used in this study was prepared by mixing a host nematic liquid crystal (5CB and JC-1041XX), a chiral dopant [ISO-(6OBA) $)_{2}$, monofunctional and difunctional UV-curable monomers (12CA and RM257), and a photoinitiator (DMPAP) at a weight ratio of 42.1:42.1:6.8:4.1:4.1:0.8. The mixture was infiltrated into liquid crystal cells assembled from two indium tin oxide 


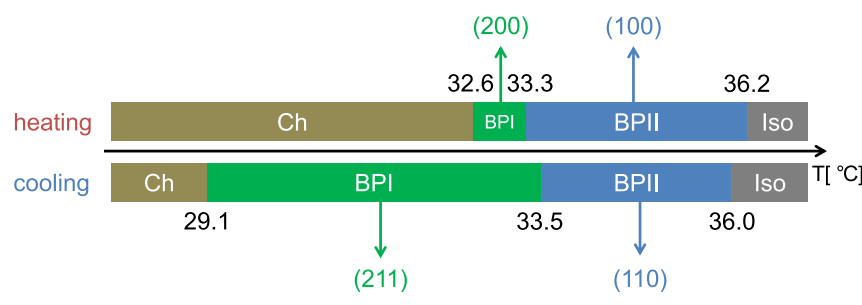

FIG. 1. (Color online) Phase sequence of the sample for heating and cooling processes before UV irradiation $\left(0.1^{\circ} \mathrm{C} / \mathrm{min}\right)$.

(ITO)-coated glass substrates with homogeneous alignment treatment (EHC Inc., KSPR-15, cell gap: $15 \mu \mathrm{m}$ ) in the isotropic phase $\left(60^{\circ} \mathrm{C}\right)$ via capillary force.

Figure 1 shows the phase sequence of the sample before polymer stabilization for heating and cooling processes, determined from polarized optical microscope (POM) observations. The temperature was scanned at a rate of $0.1^{\circ} \mathrm{C} / \mathrm{min}$ on a commercial hotplate (Linkam LTS-350). The sample exhibited BPs I and II for both heating and cooling processes; interestingly, however, the lattice plane orientation was found to be dependent on the direction of the temperature scan. The sample oriented its (200) and (100) planes parallel to the substrates in BPs I and II, respectively, when heating the sample from the cholesteric phase. On the other hand, when the sample was cooled from the isotropic phase, the sample oriented its (211) and (110) planes, respectively, for BPs I and II. The (110) orientation of BP I was obtained by cooling the sample from BP II with (100) alignment. The physical mechanism behind the rotation of the lattice is unclear but could be due to the discontinuous change in the pitch that occurs at the transition [19].

To ensure that the whole sample had the desired orientation of either (110) or (200), the sample was heated and cooled repetitively within the temperature range of BP I [20]. After confirming that the whole sample showed the desired orientation, the two samples were irradiated with UV light of intensity $1.0 \mathrm{~mW} / \mathrm{cm}^{2}$ (measured at $365 \mathrm{~nm}$ ) for $30 \mathrm{~min}$ to stabilize the structure.

The orientation of the samples was determined from the reflection POM, microspectroscopy, and Kossel pattern observations. The reflection spectra were measured using a spectrometer (Ocean Optics, USB4000) equipped with an optical fiber with diameter $550 \mu \mathrm{m}$ and coupled to a $10 \times$ objective lens, and Kossel patterns were observed at $\lambda=441 \mathrm{~nm}$ using a $100 \times$ objective lens with a numerical aperture (NA) of 0.85 . The Kerr coefficient of the samples were evaluated by measuring the change in the refractive index induced by an electric field. Because the electric field applied vertically between the substrates has little effect on the transmittance between crossed polarizers, the transmittance was measured with the analyzer removed, and the refractive index was evaluated by analyzing the interference fringes in the transmittance spectrum. As it will be shown later, spectral fits were performed over the region $600-700 \mathrm{~nm}$, since the PSBP samples showed Bragg reflection around $\sim 550 \mathrm{~nm}$. For normal incidence, the transmittance spectrum of a nonabsorbing medium placed between glass substrates varies according to the equation

$$
\frac{I_{t}}{I_{i}}=\frac{\left(1-r^{2}\right)^{2}}{\left(1-r^{2}\right)^{2}+4 r^{2} \sin ^{2}(\phi / 2)}, \phi=2 n k_{0} d,
$$

where $I_{t}$ and $I_{i}$ are the intensities of the transmitted and incident light, respectively, $\phi$ is the phase shift after one round trip, $r$ is the Fresnel reflection coefficient, $n$ is the refractive index, $k_{0}$ is $2 \pi / \lambda$, and $d$ is the cell gap. $n$ is the sole fitting parameter since $r=\left(n_{\text {ITO }}-n\right) /\left(n_{\text {ITO }}+n\right)$, where $n_{\text {ITO }}$ is the refractive index of ITO. We assumed a constant refractive index over the range $600-700 \mathrm{~nm}$, and used $n_{\text {ITO }}=1.77$, the value at $650 \mathrm{~nm}$ available from the literature [21]. Dispersion of the liquid crystal refractive index was also not considered, because dispersion is typically not so large in the wavelength region and, as we show in the results, good fits were obtained even when a constant refractive index was assumed. The change in refractive index $\delta n$ is the difference in $n$ between the field on and off states, and the birefringence is given by $\Delta n=3 \delta n$. The gradient of the induced birefringence plotted against the square of the electric field yields the Kerr coefficient according to Eq. (1) $(\lambda=650 \mathrm{~nm}$ was used to evaluate the Kerr coefficient). In practice, one often finds that the ideal quadratic relationship holds only at low applied fields and saturates at higher voltages. An extended Kerr effect formula has been developed to evaluate the Kerr coefficient in such cases:

$$
\begin{aligned}
\Delta n & =\Delta n_{\text {sat }}\left(1-\exp \left[-\left(\frac{E}{E_{\text {sat }}}\right)^{2}\right]\right), \\
K & =\Delta n_{\text {sat }} /\left(\lambda E_{\text {sat }}^{2}\right),
\end{aligned}
$$

where $\Delta n_{\text {sat }}$ is the saturation electric field-induced birefringence and $E_{\text {sat }}$ is the saturation electric field [22]. For this experiment, the transmittance spectra were measured using a spectrometer (Hamamatsu Photonics, PMA-11) equipped with an optical fiber with diameter $1 \mathrm{~mm}$ and coupled to a $50 \times$ objective lens, so that spectra from single domains could be collected. The crystal orientation of the (110)-oriented sample was controlled so that the incident light was polarized along the [1ㅣㄹ direction. From symmetry considerations, the (200)-oriented sample has no dependence on the incident polarization direction and therefore was not set to a specific direction. The samples were driven by a square-wave electric field with a frequency of $1 \mathrm{kHz}$.

\section{RESULTS AND DISCUSSION}

Figure 2 shows the POM images and reflection spectra of the (110)- and (200)-oriented PSBP samples. The micrographs were acquired at room temperature, indicating that both samples were successfully polymer stabilized. The (200)oriented sample had larger domains than the (110)-oriented sample; however, many domains were larger than $50 \mu \mathrm{m}$, thereby enabling us to perform measurements on a single domain. The peak wavelengths of Bragg reflection occurred at 549 and $413 \mathrm{~nm}$, corresponding to the green and blue textures observed between crossed polarizers. The two peaks yield lattice constants of 245 and $254 \mathrm{~nm}$, according to the equation $a=\lambda_{h k l} \sqrt{h^{2}+k^{2}+l^{2}} / 2 n$, where $\lambda_{h k l}$ is the Bragg wavelength and $h, k, l$ are the miller indices of the lattice plane. 
(a)

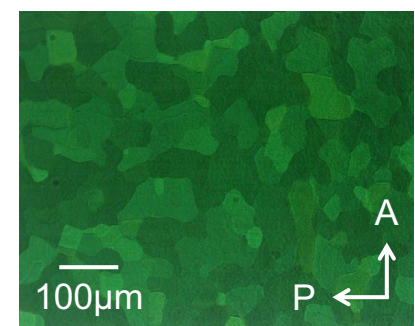

(ii)

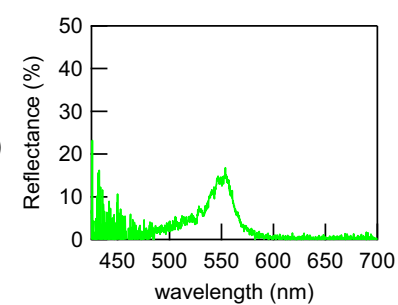

(b)
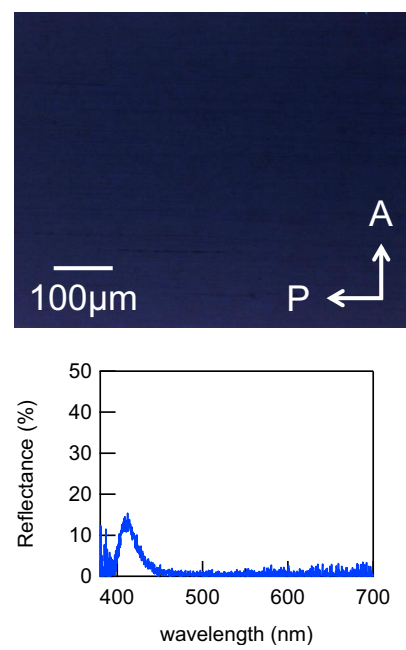

FIG. 2. (Color online) (i) POM images and (ii) reflection spectra of the (a) (110)- and (b) (200)-oriented samples through crossed polarizers after the thermal recycle process and UV irradiation.

$n$ was assumed to be 1.584 and 1.623 for the (110)- and (200)-oriented samples, respectively, by referring to measured refractive index data of the host nematic liquid crystal [23].

Figure 3 shows the Kossel patterns of the two samples. Twoand fourfold patterns were observed for the (110)- and (200)oriented samples, respectively, agreeing with the symmetry expected when a cubic crystal is viewed along the [110] and [200] directions. Furthermore, theoretical calculations of the Kossel patterns using the lattice constants obtained from the Bragg reflection peaks satisfactorily reproduce the experimental results. The orientations of the two samples are thus confirmed to be (110) and (200). The close lattice constants obtained in spite of the relatively large temperature dependence of this BP material [13] imply that high quality samples differing almost only in orientation were obtained.

Figure 4 shows examples of the transmitted spectra and fitting results. Theoretical spectral curves match well with those obtained experimentally, thus supporting the validity of our analysis. Figure 5 shows the change in electric fieldinduced birefringence plotted against the square of the applied electric field evaluated from the fitting results. The solid lines are fits to the data according to the extended Kerr formula (3) [22]. Differing behaviors were observed for the (110)and (200)-oriented samples: the refractive index of the (110)oriented sample showed a nonlinear response that gradually

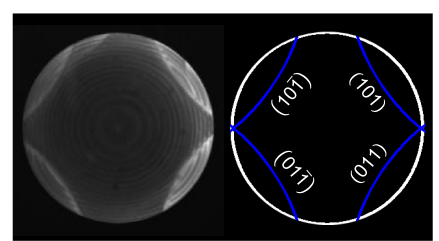

(a)

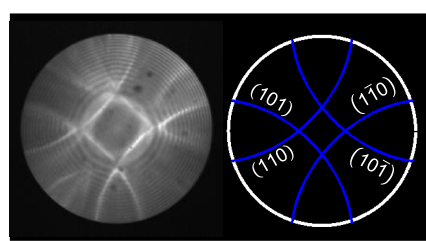

(b)
FIG. 3. (Color online) Kossel patterns and theoretical calculation results of the (a) (110)- and (b) (200)-oriented samples after the thermal recycle process and UV irradiation at room temperature.

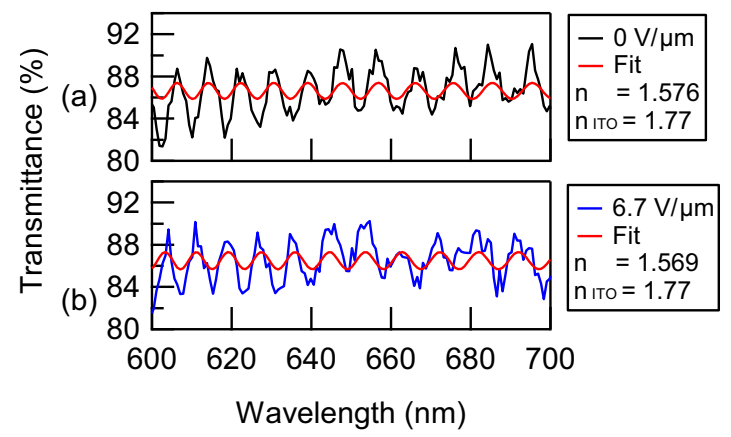

FIG. 4. (Color online) Transmitted spectra and fitting results for (a) off $(0 \mathrm{~V} / \mu \mathrm{m})$ and (b) on $(6.7 \mathrm{~V} / \mu \mathrm{m})$ states.

saturates, while the (200)-oriented sample showed a linear relationship within the range of the applied field.

Table I shows the fitting parameters used in the extended Kerr model. The experiments were performed on two (110)-oriented samples and three (200)-oriented samples, and the change in the refractive index was measured at three different positions for each sample. The sample averages of the Kerr coefficients were 0.87 and $0.72\left[\mathrm{~nm} / \mathrm{V}^{2}\right]$ for the (110)and (200)-oriented samples, indicating the Kerr coefficient of the (110)-oriented sample to be larger than that of the (200)oriented sample by $20 \%$. Moreover, the unbiased estimates of population variance for the two samples were $3.4 \times 10^{-3}$ and $7.6 \times 10^{-4}$, which yielded the following $95 \%$ confidence intervals: $0.81-0.93\left[\mathrm{~nm} / \mathrm{V}^{2}\right]$ for the (110)-oriented sample, and $0.70-0.75\left[\mathrm{~nm} / \mathrm{V}^{2}\right]$ for the (200)-oriented sample. We therefore conclude that there is a nonnegligible difference in the Kerr coefficient depending on the lattice orientation. On the other hand, both $\Delta n_{\text {sat }}$ and $E_{\text {sat }}$ of the (110)-oriented sample were smaller than that of the (200)-oriented sample, reflecting the greater saturation behavior observed in the (110)-oriented sample. It should be noted that the saturation parameters for the (200)-oriented sample, in which very small saturation was observed, are purely numerical and thus have no physical significance, i.e., it does not mean that the sample will exhibit field-induced birefringence of 0.30 . These results imply that the electro-optic response of PSBPs is different when the field

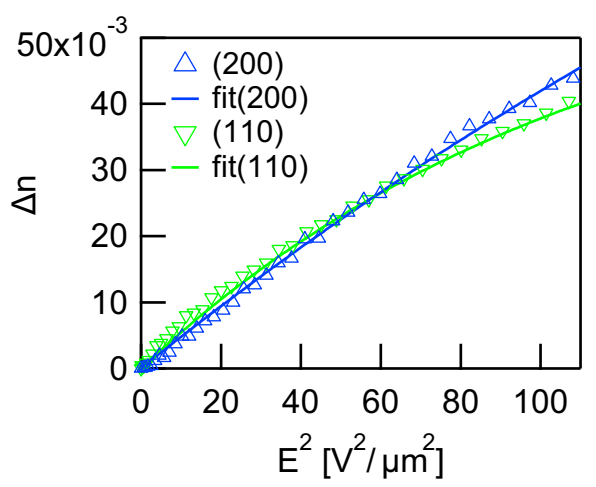

FIG. 5. (Color online) Electric field-induced birefringence plotted against the square of the applied electric field and the curve by the fitting of the extended Kerr effect formula. 
TABLE I. Fitting parameters for the extended Kerr effect model.

\begin{tabular}{lcc}
\hline \hline Parameter & $(110)$ & $(200)$ \\
\hline$K\left[\mathrm{~nm} / \mathrm{V}^{2}\right]$ & 0.87 & 0.72 \\
$\Delta n_{\text {sat }}$ & 0.081 & 0.30 \\
$E_{\text {sat }}[\mathrm{V} / \mu \mathrm{m}]$ & 11.9 & 22.9 \\
\hline \hline
\end{tabular}

is applied along the (110) and (200) directions, and that the Kerr effect is anisotropic, similar to cubic optical crystals.

We discuss the microscopic origin of the anisotropy in the Kerr effect. For simplicity, we consider a model in which the structure of BP I is described by a lattice of double-twist cylinders (DTCs) floating in an isotropic medium (Fig. 5) [24]. In a DTC, the liquid crystal molecules are oriented parallel to the cylinder axis at the center and twists by $45^{\circ}$ in the radial direction: a helical molecular arrangement is thus formed in the direction perpendicular to the cylinder axis. An applied field induces birefringence in both the DTCs and the isotropic regions, but the contribution of the isotropic region is assumed to be constant regardless of the BP lattice orientation, as the volume fraction of the isotropic region does not change with the direction of the applied field. The difference in the Kerr coefficient is, therefore, discussed based on how the orientation of the DTCs with respect to the applied field can affect the induced birefringence. As can be seen from Fig. 6, the (110)-oriented sample contains 2 DTCs with perpendicular and 4 DTCs with oblique $\left(45^{\circ}\right)$ orientations to the field, while the (200)-oriented sample contains 4 DTCs perpendicular and 2 DTCs parallel to the field.

The response of a DTC to an applied field can be understood by considering how the helical molecular arrangement constituting the DTC responds to an electric field. When a field is applied parallel to the helical axis of a helical molecular arrangement, the liquid crystal molecules do not move until a threshold is reached, and reorient abruptly once the threshold is reached, in a manner similar to the Frederiks transition in nematic liquid crystals [25]. The threshold is related to the bend elastic constant $K_{33}$, helical pitch $p_{0}$, dielectric anisotropy $\epsilon_{a}$ of the liquid crystal, and dielectric constant of vacuum $\epsilon_{0}$ through the expression $E_{c}=2 \pi \sqrt{K_{33}} / p_{0} \sqrt{\epsilon_{0} \epsilon_{a}}$, and by using values $K_{33}=12.1[\mathrm{pN}], p_{0}=250[\mathrm{~nm}], \epsilon_{a}=8.85$,

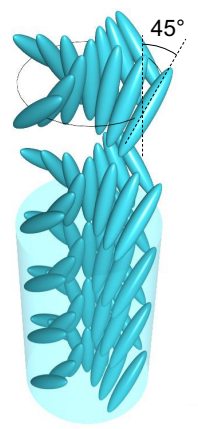

(a)

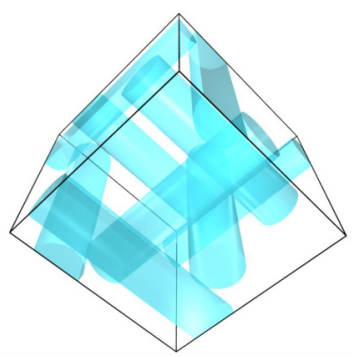

(b)

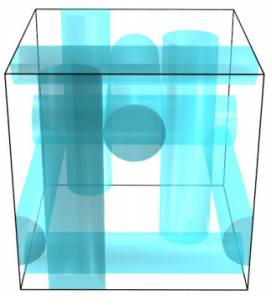

(c)
FIG. 6. (Color online) The model of (a) the double twisted cylinder and the BP I unit cells as the (b) (110) and (c) (200) planes oriented along the direction of the applied field.

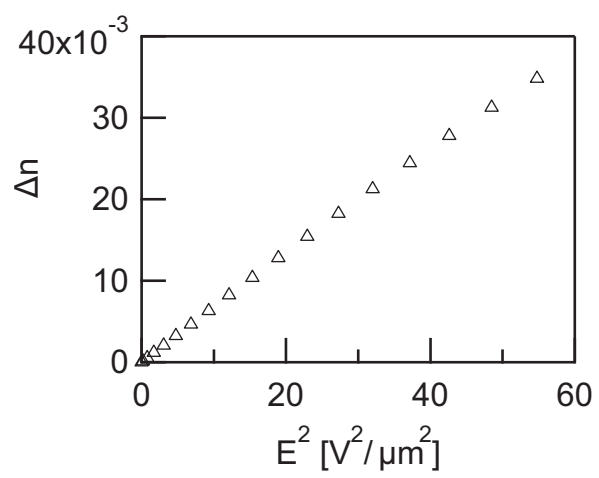

FIG. 7. Change in the field-induced birefringence of the cholesteric liquid crystal perpendicular to the applied electric field.

and $\epsilon_{0}=8.85 \times 10^{-12}[\mathrm{~F} / \mathrm{m}], \quad E_{c}$ is evaluated to be $10.1[\mathrm{~V} / \mu \mathrm{m}][9,23]$. This threshold is significantly higher than the region where the ideal quadratic relationship between the induced birefringence and the applied field is observed in experiment; the contribution of this mode to the field-induced birefringence is, therefore, assumed to be zero in this analysis. On the other hand, an electric field applied perpendicular to the helix axis gradually unwinds the helix with roughly a quadratic dependence on the field intensity [26]. By minimizing the elastic free-energy in a one-dimensional helical system, it is possible to numerically calculate the induced birefringence for this mode. Figure 7 shows the dependence of the induced birefringence on the square of the field intensity, calculated using parameters $K_{22}=3.81[\mathrm{pN}], n_{o}=1.52, n_{e}=1.68$, $\epsilon_{a}=8.85$, and $p_{0}=250[\mathrm{~nm}]$, where $K_{22}$ is the twist elastic constant (see the Appendix for the details of the calculation). The induced birefringence has a quadratic dependence on the field intensity, implying that this response is observed as an Kerr-type electro-optic effect.

The discussion above tells us that the DTCs with their axes oriented parallel to the electric field have the highest contribution to the Kerr coefficient, followed by those that are aligned at $45^{\circ}$, and finally those that are perpendicular to the field. For the helices in the DTCs aligned obliquely and perpendicular to an electric field, the effective electric field that exerts the torque to unwind the helix varies as $\cos (\theta / 2)$ and $\cos \theta$, where $\theta$ is the angle the helical axis makes with the electric field. Moreover, the contribution to the refractive index also varies as $\cos (\theta / 2)$ and $\cos \theta$. Therefore, assuming that the contribution of the DTC with its axis aligned parallel to the field, $C_{\text {para }}$, is 1 , the contributions of the oblique and perpendicular DTCs $\left(C_{\mathrm{obl}}\right.$ and $\left.C_{\text {perp }}\right)$ are calculated to be

$$
\begin{aligned}
C_{\text {obl }} & =\frac{4 \int_{0}^{\pi / 2} \cos ^{3}(\theta / 2) d \theta}{4 \int_{0}^{\pi / 2} d \theta}=0.75, \\
C_{\text {perp }} & =\frac{4 \int_{0}^{\pi / 2} \cos ^{3} \theta d \theta}{4 \int_{0}^{\pi / 2} d \theta}=0.42 .
\end{aligned}
$$

The field-induced birefringence for the (110)- and (200)oriented samples is evaluated by multiplying the following 
factors by the field-induced birefringence calculated for a cholesteric liquid crystal (Fig. 7), where $\pi / 64$ is the volume fraction of a single DTC in a unit cell:

$$
\begin{aligned}
& C_{110}=\frac{\pi}{64}\left\{4 C_{\text {obl }}+2 C_{\text {perp }}\right\}=0.188, \\
& C_{200}=\frac{\pi}{64}\left\{4 C_{\text {perp }}+2 C_{\text {para }}\right\}=0.181 .
\end{aligned}
$$

The results tells us that the Kerr coefficient of the (110)oriented sample is 1.04 times larger than that of the (200)oriented sample. A real PSBP is different from the one modeled here in that polymer networks exist between the DTCs and, although we have assumed that each DTC responds independently to an electric field, the DTCs are elastically connected, since the region assumed to be isotropic is in reality filled with liquid crystalline molecules. We consider that the numerical simulation based on the Landau-de Gennes theory is necessary to clarify the detailed electro-optic response. However, even with this simplified analysis, results qualitatively agreeing with experimental observations are obtained, that the Kerr coefficient is anisotropic, and its magnitude is larger when the field is applied along the [110] direction.

The difference in the saturation behavior is something that cannot be explained by the analysis presented above. Yan et al. have suggested that the saturation behavior originated in molecular anchoring imposed by the polymer network on the mobile liquid crystal (LC) molecules [22]. Because the two samples investigated in this study were fabricated using the same material and polymerization conditions (implying similar polymer networks exist in both samples), the results imply that not only the Kerr coefficient but the effect of molecular anchoring is dependent on the direction of the applied field. An alternative explanation is that a nonlinear effect emerges as a result of the difference in how the helices within the DTCs respond when an electric field is applied in different directions. Testing of these hypotheses requires further experiments, but should be possible, for example, by observing the polymer network in real space or investigating PSBP samples with other lattice plane orientations.

\section{CONCLUSION}

In conclusion, we investigated the Kerr effect of PSBPs with (110) and (200) planes oriented parallel to the substrates. Both the Kerr coefficient and saturation behavior were found to be dependent on the direction of the applied electric field, with the Kerr coefficient of the (110)-oriented sample being greater than the (200)-oriented sample by $20 \%$. The fact that the Kerr effect of PSBPs depends on the lattice orientation may pose issues in certain applications, such as polarization-independent tunable lenses and filters [27,28]. It may also impact the research and development of PSBP-based displays [29], although its influence may be different when the field is applied in the cell-planar direction. Our work suggests that, contrary to the common understanding that alignment is unnecessary for the electro-optic application of BPs, alignment is in fact important.

\section{ACKNOWLEDGMENTS}

This work was partly supported by Japan Society for the Promotion of Science (JSPS) of Japan KAKENHI Grants No. 24656015, No. 23656221, and No. 23107519, the Japan Science and Technology Agency (JST) of Japan PRESTO Program, and the Photonic Advanced Research Center (PARC) of Osaka University. We also thank JNC Corporation for kindly providing of the nematic liquid crystal material.

\section{APPENDIX: ELECTRO-OPTIC RESPONSE OF A CHOLESTERIC LIQUID CRYSTAL WITH FIELD APPLIED PERPENDICULAR TO THE HELIX}

Assuming the helical axis to exist along the $y$ axis, the distribution of the liquid crystal "director," which is the direction in which the constituent molecules are oriented on average, is given by $n_{x}(y)=\sin \theta(y), n_{y}(y)=0$, and $n_{z}(y)=\cos \theta(y)$, where $\theta(y)=2 \pi y / p_{0}$ and $p_{0}$ is the pitch (the distance over which the molecules rotate by $2 \pi$ ). When an electric field is applied in the $z$ direction, the free energy (Frank elastic energy) of the system is described by

$$
f=\frac{1}{2} K_{22}\left(\theta^{\prime}-q_{0}\right)^{2}+\frac{1}{2} \epsilon_{a} \epsilon_{0} E^{2} \sin ^{2} \theta+\text { constant }
$$

where $K_{22}$ is the twist elastic constant, $\theta^{\prime}=\partial \theta / \partial y$, and $q_{0}=$ $2 \pi / p_{0}$ [25]. The constant in this equation does not affect the director configuration and can be omitted. Using the EulerLagrange equation to minimize the free energy, we obtain

$$
\begin{gathered}
\frac{d \theta}{d y}=q_{0}\left[\left(\frac{\pi E}{2 E_{0}} \sin \theta\right)^{2}+A\right]^{1 / 2}, \\
E_{0}=\frac{\pi^{2}}{p_{0}} \sqrt{\frac{K_{22}}{\epsilon_{0} \epsilon_{a}}},
\end{gathered}
$$

where $A$ is the integration constant. Because the pitch of a PSBP is not changed by an electric field, we assume that the pitch is invariant [30]. In this case, the value of $A$ can be found by solving the equation

$$
\int_{0}^{\pi}\left[\left(\frac{\pi E}{2 E_{0}} \sin \theta\right)^{2}+A\right]^{-1 / 2} d \theta=\pi .
$$

The director distribution is converted to the dielectric tensor distribution via the relationship

$$
\begin{aligned}
& {\left[\begin{array}{lll}
\varepsilon_{x x} & \varepsilon_{x y} & \varepsilon_{x z} \\
\varepsilon_{y x} & \varepsilon_{y y} & \varepsilon_{y z} \\
\varepsilon_{z x} & \varepsilon_{z y} & \varepsilon_{z z}
\end{array}\right]} \\
& =\left[\begin{array}{ccc}
n_{o}^{2} \cos ^{2} \theta+n_{e}^{2} \sin ^{2} \theta & 0 & \left(n_{e}^{2}-n_{o}^{2}\right) \cos \theta \sin \theta \\
0 & n_{0}^{2} & 0 \\
\left(n_{e}^{2}-n_{o}^{2}\right) \cos \theta \sin \theta & 0 & n_{o}^{2} \sin ^{2} \theta+n_{e}^{2} \cos ^{2} \theta
\end{array}\right],
\end{aligned}
$$

where $n_{e}$ and $n_{o}$ are the extraordinary and ordinary refractive indices. Taking the average over one pitch and calculating $\Delta n=\sqrt{\epsilon_{x x}}-\sqrt{\epsilon_{z z}}$ for each field intensity yields Fig. 7. 
[1] D. C. Wright and N. D. Mermin, Crystalline liquids: the blue phases, Rev. Mod. Phys. 61, 385 (1989).

[2] J. Yan, S.-T. Wu, K.-L. Cheng, and J.-W. Shiu, A full-color reflective display using polymer-stabilized blue phase liquid crystal, Appl. Phys. Lett. 102, 081102 (2013).

[3] M. Kimura, N. Nagumo, T. N. Oo, N. Endo, H. Kikuchi, and T. Akahane, Single-substrate polymer-stabilized blue phase liquid crystal display, Opt. Mater. Express 3, 2086 (2013).

[4] H. Kikuchi, M. Yokota, Y. Hisakado, H. Yang, and T. Kajiyama, Polymer-stabilized liquid crystal blue phases, Nat. Mater. 1, 64 (2002).

[5] T. Iwata, K. Suzuki, N. Amaya, H. Higuchi, H. Masunaga, S. Sasaki, and H. Kikuchi, Control of cross-linking polymerizaion kinetics and polymer aggregated structure in polymerstabilized liquid crystalline blue phases, Macromolecules $\mathbf{4 2}$, 2002 (2009).

[6] J. Kerr, XL. A new relation between electricity and light: Dielectrified media birefringent, Philos. Mag. 50, 337 (1875).

[7] T. Iwata, K. Suzuki, H. Higuchi, and H. Kikuchi, A method for enlarging the Kerr constant of polymer-stabilised blue phases, Liq. Cryst. 36, 947 (2009).

[8] Y. Hisakado, H. Kikuchi, T. Nagamura, and T. Kajiyama, Large electro-optic Kerr effect in polymer-stabilized liquid-crystalline blue phases, Adv. Mater. 17, 96 (2005).

[9] L. Rao, J. Yan, S.-T. Wu, S. Yamamoto, and Y. Hasebe, A large Kerr constant polymer-stabilized blue phase liquid crystal, Appl. Phys. Lett. 98, 081109 (2011).

[10] Y. Chen and S.-T. Wu, The outlook for blue-phase LCDs, Proc. SPIE 9005, 900508 (2014).

[11] J. Yan and S.-T. Wu, Polymer-stabilized blue phase liquid crystals: a tutorial [Invited], Opt. Mater. Express 1, 1527 (2011).

[12] Y. Chen and S.-T. Wu, Recent advances on polymer-stabilized blue phase liquid crystal materials and devices, J. Appl. Polym. Sci. 131, 40556 (2014).

[13] H. Choi, H. Higuchi, and H. Kikuchi, Electrooptic response of liquid crystalline blue phases with different chiral pitches, Soft Matter 7, 4252 (2011)

[14] H. Tone, H. Yoshida, S. Yabu, M. Ozaki, and H. Kikuchi, Effect of anisotropic lattice deformation on the Kerr coefficient of polymer-stabilized blue-phase liquid crystals, Phys. Rev. E 89, 012506 (2014).

[15] J. F. Nye, Physical Properties of Crystals (Clarendon Press, Oxford, 1985).

[16] F. Porsch, H. Stegemeyer, and K. Hiltrop, Electric field-induced birefringence in liquid-crystalline blue phases, Z. Naturforsch 39a, 475 (1984).
[17] H.-S. Kitzerow, Blue phases come of age: a review, Proc. SPIE 7232, 723205 (2009).

[18] H.-S. Kitzerow, Blue phases: prior art, potential polar effects, challenges, Ferroelectrics 395, 66 (2010).

[19] H. Stegemeyer, Helical pitch change at the phase transition blue phase to cholesteric, Phys. Lett. A 79, 425 (1980).

[20] H.-S. Chen, Y.-H. Lin, C.-H. Wu, M. Chen, and H.-K. Hsu, Hysteresis-free polymer-stabilized blue phase liquid crystals using thermal recycles, Opt. Mater. Express 2, 1149 (2012).

[21] T. A. F. Konig, P. A. Ledin, J. Kerszulis, M. A. Mahmoud, M. A. El-Sayed, J. R. Reynolds, and V. V. Tsukruk, Electrically tunable plasmonic behavior of nanocube-polymer nanomaterials induced by a redox-active electrochromic polymer, ACS Nano 8, 6182 (2014).

[22] J. Yan, H.-C. Cheng, S. Gauza, Y. Li, M. Jiao, L. Rao, and S.-T. Wu, Extended Kerr effect of polymer-stabilized blue-phase liquid crystals, Appl. Phys. Lett. 96, 071105 (2010).

[23] J. B. Lias, H. Kikuchi, M. Kimura, and T. Akahane, Determination of polar anchoring strength for polymer-stabilized blue phase liquid crystal device, Jpn. J. Appl. Phys. 50, 081607 (2011).

[24] C. Bohley and T. Scharf, Polarization of light reflected by cholesteric blue phases, J. Opt. A 6, S77 (2004)

[25] D.-K. Yang and S.-T. Wu, Fundamentals of Liquid Crystal Devices (Wiley, New York, 2006).

[26] P. P. Crooker, Chirality in Liquid Crystals, edited by H.-S. Kitzerow and C. Bahr (Springer-Verlag, New York, 2001).

[27] Y.-H. Lin, H.-S. Chen, H.-C. Lin, Y.-S. Tsou, H.-K. Hsu, and W.-Y. Li, Polarizer-free and fast response microlens arrays using polymer-stabilized blue phase liquid crystals, Appl. Phys. Lett. 96, 113505 (2010).

[28] Y.-H. Chen, C.-T. Wang, C.-P. Yu, and T.-H. Lin, Polarization independent Fabry-Pérot filter based on polymer-stabilized blue phase liquid crystals with fast response time, Opt. Express 19, 25441 (2011).

[29] Z. Ge, S. Gauza, M. Jiao, H. Xianyu, and S.-T. Wu, Electrooptics of polymer-stabilized blue phase liquid crystal displays, Appl. Phys. Lett. 94, 101104 (2009).

[30] H. Yoshida, S. Yabu, H. Tone, Y. Kawata, H. Kikuchi, and M. Ozaki, Secondary electro-optic effect in liquid crystalline cholesteric blue phases, Opt. Mater. Express 4, 960 (2014). 